Available online on 15.05 .2015 at http://jddtonline.info
Journal of Drug Delivery and Therapeutics
Open access to Pharmaceutical and Medical research
(C) 2015, publisher and licensee JDDT, This is an Open Access article which permits unrestricted noncommercial use, provided the original
work is properly cited

\title{
RESEARCHARTICLE
}

\section{ETHNOBOTANICAL SURVEY OF PLANTS USED IN THE MANAGEMENT OF DIABETES MELLITUS IN ABEOKUTA, NIGERIA}

\author{
${ }^{1}$ Kadiri M, ${ }^{2}$ Ojewumi AW, ${ }^{3}$ Agboola DA, ${ }^{4}$ Adekunle MF \\ ${ }^{1-3}$ Department of Pure and Applied Botany, Federal University of Agriculture, Abeokuta, Ogun State Nigeria \\ ${ }^{4}$ Department of Forestry and Wild life Management, Federal University of Agriculture, Abeokuta, Ogun State Nigeria \\ *Corresponding Author's E-mail: mukailakadiri@yahoo.com
}

Received 20 Feb 2015; Review Completed 03 April 2015; Accepted 08 April 2015, Available online 15 May 2015

\begin{abstract}
An ethnobotanical survey of some plants used for management of diabetes in Abeokuta, Ogun State, Nigeria was conducted. A total of 100 questionnaires were administered; (50 herbal practitioners and 50 on patients). Investigations were carried out on the plant parts used, methods of preparation and administration including, dosage and duration of usage. The results showed that fifty (50) plant species belonging to 30 families used in herbal anti-diabetes recipes. The most frequently used plant was Vernonia amygdalina (54\%), followed by Azadirachta indica (44\%), Ocimum gratissimum (20\%), Mormodica charantia (10\%) and Citrus aurantifolia (10\%). Irrespective of the plant parts (leaves, fruits, stem- barks or roots) or combinations of the plant parts, water and alcohol were the main solvents. The most frequently used plant parts was leaf (62\%) and mainly by decoction. Treatment regimens were by chewing or drinking of the aqueous herbal preparations (350-400ml) daily for 6-12 weeks or until symptoms of diabetes disappear.
\end{abstract}

Keywords: Anti-diabetic plants, ethnobotanical survey, herbs, plant parts.

\section{INTRODUCTION}

In Africa, the number of people suffering from diabetes has been rising steadily over the past two decades. Several reports have stressed the high mortality from diabetes especially among the blacks (Durmuskahya and Özturk, 2013).There are nearly 285 million (6.6\% of population aged 20-79 years) diabetic patients across the globe. In 2005, it was reported that nearly 1.1 million people died worldwide due to this disease. It is estimated that the number of diabetes patients will reach 450 million in 2030 with $97 \%$ showing type 2 diabetes mellitus (T2DM; non-insulin dependent diabetes mellitus) if positive actions are not put in place (Israili 2010, Israili, 2011; Durmuskahya and Özturk, 2013). Diabetes mellitus is a disease of metabolic impairment of carbohydrate, fat and protein due to either lack of insulin secretion or decreased sensitivity of the tissues to insulin. It is characterized by severe socio-economic importance such as hyperglycemia and glycosuria (Aguwa et al., 2004). Diabetes mellitus and its associated complications have significant impact on health, quality of life and life expectancy of its sufferers.

Several findings indicated that traditional medicine is patronized by all segments of the society because it is affordable health care delivery system of people (Soladoye et al., 2012; Aslan et al., 2010; Inanç et al., 2007; Onal et al. 2005; Ozbek et al., 2004).
Many drugs have been developed and used globally in managing hyperglycemia in type 2 diabetes mellitus (Israili, 2011). However, therapy becomes less effective over time as more than $50 \%$ of patients do not achieve normal glycemic goals (Israili, 2010). Also, the few conventional drugs are characterized by appreciable level of toxicity, high cost and non availability. Thus, people seek relief from traditional healing which provides measures for the treatment of the disease (Farnsworth et al., 1985; Morris, 2002). This is due to the general roles of plants in disease management as biological potentials (glibenclamide). Herbal medicine is used by more than $50 \%$ of the world population. In India $65 \%$ of population have used traditional medicine for management of several ailments (Pratul , 2011). A recent survey has revealed that 35 to $41 \%$ of diabetic patients use complementary and alternative medicines (mostly botanicals) in addition to conventional medicine (Ceylan et al., 2009; Küçükgüçlü et al., 2010). However, there has been increasing demand for the use of plant products with anti-diabetic activity due to low cost, easy availability, abundance and lesser side effects. Plant materials are continuously scrutinized and explored for their effect as hypoglycemic agents. The objective of the present study was to identify some plants used in the treatments of diabetes. This study was carried out to document ethnobotanical information of indigenous plants used to manage of diabetes mellitus in Abeokuta 


\section{MATERIALS AND METHODS}

\section{The Study Area}

The study was conducted in Abeokuta, the capital city of Ogun State, South West Nigeria. A total of 10 Markets including Lafenwa, Odeda, Osiele, Kuto, Iberekodo, Elega, Alabata, Olodo, Itoku, Omida and various herbal shops of Abeokuta were visited.

The town has a population of about one million people who are mainly civil servants and traders. It is predominantly dominated by the Egbas with sizable proportion of Yewas, Ijebu and other Yoruba ethnic groups in Nigeria. There are two main Local Government Areas in Abeokuta: Abeokuta North and Abeokuta South. Abeokuta is in the tropical rain forest zone of South Western Nigeria but the vegetation now is that of derived savannah. The Nation's main air port, sea port and industrial centre, Lagos is 104 kilometers to the south while Ibadan the largest city in the country is 77 kilometers to the North. Abeokuta falls within the longitude $3^{\circ} 21^{\prime}$ East and latitude $7^{\circ} 11^{\prime}$ "North. The annual temperature range from $22.8^{0}$ to $34.9^{\circ} \mathrm{C}$. The mean annual rainfall is about $107 \mathrm{~mm}$ (Adekunle and Akinlemibola, 2008).

\section{Informed consent}

The purpose of the study was explained to the local traditional practitioners, herb sellers, community leaders, market-head leaders and herbal shop owners in the town. Consent to was given by the head of traditional herb practitioners and community leaders. Informed consent was obtained from each of the participants. An approval for the study was obtained from market leaders and traditional heads of the communities in which the markets were located.

\section{General questionnaire}

A total of 100 structured questionnaire were administered using stratified method on traditional herbal practitioners, herb sellers and diabetic patients, to obtain information on commonly used herbs, names of plants, parts used, methods of herbal preparation, route of administration, mode of administration, dosage measurement and duration of usage. The questionnaires were administered by systematic random sampling method in such a way that only every fifth numbered respondent was chosen.

\section{RESULTS}

\section{Socio-Economic Characteristics of Respondents}

\section{Gender. Age and education status}

Of 100 questionnaires administered; 50 was to herbal practitioners who included traditional healers and herbal sellers and the other 50 to the patients (table 1). Larger number of (Traditional Herbal Practitioners) (THP); 28 representing $56 \%$ of the total traditional healers and herbal sellers were males while $22(44 \%)$ of the respondents were females (table 1 ).

$46 \%$ and $40 \%$ of the total population of the Traditional herbal practitioners and patients respectively were between the age group of 41-50 years, $4 \%$ was recorded at 20-30 years while at 61-70 years, $6 \%$ of the (c) 2011-15, JDDT. All Rights Reserved respondents was reported (table 1). Similarly, at age 41$50,34 \%$ of the Traditional herbal practitioners were males while $12 \%$ were females. At age 40-50 years, $14 \%$ of patients were males while $26 \%$ were female. Meanwhile at age 20-30 years, equal percentage of male and female $(2 \%)$ traditional herbal practitioners were reported. Although both males and females engaged in traditional herbal practices, the percentage of males $(74 \%)$ was higher than female $(26 \%)$ and males were less affected by diabetes ( $42 \%$ ) than females $(58 \%)$.

The educational status of respondents (THP) and patients showed that 4 traditional herbal practitioners and 16 patients representing $8 \%$ and $32 \%$ of the total had no formal education. Majority of THP were West African Examination Council certificate holders as represented by $42 \%$ while majority of patients were illiterates as represented by $32 \%$ (Table 1). This study revealed a total of fifty (50) species of plants belonging to 30 families as being used in the control and treatment of diabetes in Abeokuta. An ethnobotanical data of the identified plants were summarized in Table 2. 31(62\%) plant species with leaves being used were Aframomum melgueta, Azadirachta indica, Ageratum conyzoides, Alstonia boonei, Aloe vera, Blighia sapida, Cassia siamea, Carica papaya, Crudia klainei, Cymbopogon citrates, Ficus asperifolia, Glyphaea brevis, Hibiscus sabdariffa, Terminalia schimperiana, Jatropha curcas, Lawsonia inermis, Mangifera indica, Mormodica indica, Morinda lucida, Nicotiana tabacum, Ocimum gratissimum, Phyllantus amarus, Bambus vulgaris, Gossipium spp, Rauwolfia vomitoria, Solanum aethiopicum, Sida acuta, Senna podocarpa, Tridax procumbens, Uvaris afzelii and Vernonia amygdalina. $5(10 \%)$ of the plants were prepared using roots naming Alstonia congensis, Garcinia kola, Securidaca longepedunculata, Senecio biafrae, Tetrapleura tetraptera. Cucurneropsis mannii, Syzygium guineense and Bidens pilosa. Allium sativum and Allium cepa used bulbs (4\%). Musa nana and Musa parasiadiaca had stem (Culm). Bark from Anacardium occidentale was used, representing (2\%). Rhizomes from Zingiber officinale (2\%) while leaf/root and leaf/ bark combinations were represented as parts being used by 2 plants (4\%) (Table2). The most frequently utilized plants were Vernonia amygdalina (54\%), Azadirachta indica (44\%), Ocimum gratissimum (20\%), Mormodica charantia (10\%) and Citrus aurantifolia (10\%). Leaf (Anacardium occidentals), root (Tetrapleura tetraptera), seed (Bidens pilosa), stem (Garcinia kola), bulb (Allium sativum), rhizome (Zingiber officinale) and fruit (Citrus aurantifolia) (Table 3). The preparations were measured by the patients with either glass cup (350-400ml) and taken orally 2-3 times daily for 6-12 weeks (Table 2).

The life forms of plants covered by this study ranges from trees to herbs. $32 \%$ was trees, $14 \%$ was shrubs, $2 \%$ was climbers while $52 \%$ was herbs (table 4 ). $46 \%$ of the plants were cultivated, $36 \%$ were wild while $18 \%$ were both cultivated and wild

The preparations were decoction (46\%), juice (14\%), maceration (12\%), essence (10) juice or powder (14\%) with the least being infusion (4\%) (Table 4). 
Majority of the respondents showed preference to aqueous preparation $(64 \%)$, some mixed the preparations with pap (12\%), alcohol (12\%), lime (4\%), honey (4\%) and bile of cow (2\%) while others made use of the plants as chewing sticks for easy administration $(2 \%)$. It was observed that the herbal preparations were reported as being measured with the use of cup (76\%), tea spoons $(22 \%)$, and tablets $(2 \%)$. Some of the THPs recommended other remedies such as beans and its derivatives $(26 \%)$, vegetables and fruits $(60 \%)$, No diet (10\%) while others non- plant materials(4\%) such as potash, honey, alum etc(table4). The duration of usage was month $(72 \%)$, week $(20 \%)$ and the year $(8 \%)$ before the symptoms of the diseases such as frequent urination, weight loss and fatigue disappear(table 4). Majority of the respondents preferred mainly drinking $(350-400 \mathrm{ml})$ of the preparations 2 to 3 times daily.

Table 1: Demographic structure of the respondents (traditional healers and herbal material sellers) sampled in Abeokuta, Ogun State Nigeria

\begin{tabular}{|l|l|l|l|l|l|}
\hline $\begin{array}{l}\text { Socio-demographic data } \\
\text { (Sex) }\end{array}$ & $\begin{array}{l}\text { Traditional healers } \\
\text { (Frequency) }\end{array}$ & $\begin{array}{l}\text { Herbal material } \\
\text { sellers (Frequency) }\end{array}$ & Total & \% Total & Mode \\
\hline Male & 23 & 5 & 28 & 56 & 56 \\
\hline Female & 10 & 12 & 22 & 44 & \\
\hline Total & 33 & 17 & & 100.00 & \\
\hline Age Class (Herbal practitioners) (Years) & (Male) & (Female) & & & \\
\hline $20-30$ & 1 & 1 & 2 & 4 & \\
\hline $31-40$ & 10 & 4 & 14 & 28 & \\
\hline $41-50$ & 17 & 6 & 23 & 46 & 46 \\
\hline $51-60$ & 6 & 1 & 7 & 14 & \\
\hline $61-70$ & 3 & 1 & 4 & 8 & \\
\hline Total & 37 & 13 & & 100.00 & \\
\hline Age Class (Patients) (Years) & $($ Male) & $($ Female) & & & \\
\hline $20-30$ & 3 & 2 & 5 & 1 & \\
\hline $31-40$ & 4 & 5 & 9 & 18 & \\
\hline $41-50$ & 7 & 13 & 20 & 40 & 40 \\
\hline $51-60$ & 6 & 7 & 13 & 26 & \\
\hline $61-70$ & 1 & 2 & 3 & 6 & \\
\hline Total & 21 & 29 & & 100.00 & \\
\hline Education level (THP) & & & & & \\
\hline Illiterates & 1 & 3 & 4 & 8 & \\
\hline Primary School & 9 & 6 & 15 & 30 & \\
\hline Secondary school & 12 & 9 & 21 & 42 & 42 \\
\hline Tertiary & 7 & 3 & 10 & 20 & \\
\hline Total & 29 & 21 & & 100.00 & \\
\hline
\end{tabular}

Table 2: Some plants species used in treating diabetes in Abeokuta, Ogun State, Nigeria

\begin{tabular}{|c|c|c|c|c|c|}
\hline Family/botanical name & Common/local name & Part used & Habit & $\begin{array}{l}\text { Route of } \\
\text { administration }\end{array}$ & $\begin{array}{l}\text { Frequency of } \\
\text { mention }(\%)\end{array}$ \\
\hline \multicolumn{6}{|l|}{ Alliceae } \\
\hline Allium cepa & $\begin{array}{l}\text { Onion } \\
\text { Alubosa }\end{array}$ & Bulb & $\mathrm{HC}$ & Oral & $1(2 \%)$ \\
\hline \multicolumn{6}{|l|}{ Alliaceae } \\
\hline Allium sativum & $\begin{array}{l}\text { Garlic } \\
\text { Ayu }\end{array}$ & Bulb & $\mathrm{HC}$ & Oral & $1(2 \%)$ \\
\hline \multicolumn{6}{|l|}{ Anacardiaceae } \\
\hline Anacardium occidentale & $\begin{array}{l}\text { Cashew } \\
\text { Kaju }\end{array}$ & $\begin{array}{l}\text { Leaves } \\
\text { /barks }\end{array}$ & $\mathrm{TC}$ & Oral & $1(2 \%)$ \\
\hline \multicolumn{6}{|l|}{ Anacardiaceae } \\
\hline Mangifera indica & $\begin{array}{l}\text { Mango } \\
\text { Mangoro }\end{array}$ & Leaves & T C & Oral & $4(8 \%)$ \\
\hline \multicolumn{6}{|l|}{ Annonaceae } \\
\hline Uvari afzelii & $\begin{array}{l}\text { Monkey finger } \\
\text { Gbogbonise }\end{array}$ & Leaves & $\mathrm{H} \mathrm{W}$ & Oral & $3(6 \%)$ \\
\hline \multicolumn{6}{|l|}{ Apocynaceae } \\
\hline Alstonia boonei & Stool - wood & Leaves & $\mathrm{T}, \mathrm{W}$ & Oral & $1(2 \%)$ \\
\hline
\end{tabular}




\begin{tabular}{|c|c|c|c|c|c|}
\hline & Awogba-arun & & & & \\
\hline \multicolumn{6}{|l|}{ Apocynaceae } \\
\hline Alstonia congensis & $\begin{array}{l}\text { Stool wood } \\
\text { Ahun }\end{array}$ & Root & $\mathrm{T}, \mathrm{W}$ & Oral & $1(2 \%)$ \\
\hline \multicolumn{6}{|l|}{ Apocynaceae } \\
\hline Saccharum officinarum & $\begin{array}{l}\text { Sugar cane } \\
\text { Ireke }\end{array}$ & $\begin{array}{l}\text { Root/ } \\
\text { Leaves }\end{array}$ & $\mathrm{G}, \mathrm{C}$ & Oral & $2(4 \%)$ \\
\hline \multicolumn{6}{|l|}{ Apocynaceae } \\
\hline Rauwolfia vomitoria & $\begin{array}{l}\text { Swizzle stick } \\
\text { Asofeyeje }\end{array}$ & Leaves & $\mathrm{T}, \mathrm{C} / \mathrm{W}$ & Oral & $4(8 \%)$ \\
\hline \multicolumn{6}{|l|}{ Asphodelaceae } \\
\hline Aloe vera & Aloe & & $\mathrm{H}, \mathrm{C}$ & Oral & $1(2 \%)$ \\
\hline \multicolumn{6}{|l|}{ Asteraceae } \\
\hline Ageratum conyzoides & $\begin{array}{l}\text { Goat weed } \\
\text { Imi-esu }\end{array}$ & Leaves & $\mathrm{H}, \mathrm{W}$ & Oral & $1(2 \%)$ \\
\hline \multicolumn{6}{|l|}{ Asteraceae } \\
\hline Senecio biafrae & $\begin{array}{l}\text { Bologi } \\
\text { Worowo }\end{array}$ & Root & $\mathrm{H}, \mathrm{W}$ & Oral & $1(2 \%)$ \\
\hline \multicolumn{6}{|l|}{ Asteraceae } \\
\hline Tridax procumbens & $\begin{array}{l}\text { Tridax } \\
\text { Igbalode }\end{array}$ & Leaves & $\mathrm{H}, \mathrm{W}$ & Oral & $2(4 \%)$ \\
\hline \multicolumn{6}{|l|}{ Asteraceae } \\
\hline Vernonia amygdalina & $\begin{array}{l}\text { Bitter leaf } \\
\text { Ewuro }\end{array}$ & Leaves & $\mathrm{S}, \mathrm{C} / \mathrm{W}$ & Oral & $27(54 \%)$ \\
\hline \multicolumn{6}{|l|}{ Bromeliaceace } \\
\hline Ananas comosus & $\begin{array}{l}\text { Pineapple } \\
\text { Opon-oyinbo }\end{array}$ & Fruits & $\mathrm{H}, \mathrm{C}$ & Oral & $1(2 \%)$ \\
\hline \multicolumn{6}{|l|}{ Caesalpiniaceace } \\
\hline Senna podocarpa & $\begin{array}{l}\text { Sennas } \\
\text { Asunwo }\end{array}$ & Leaves & $\mathrm{H}, \mathrm{W}$ & Oral & $3(6 \%)$ \\
\hline \multicolumn{6}{|l|}{ Caricaceae } \\
\hline Carica papaya & $\begin{array}{l}\text { Pawpaw } \\
\text { Ibepe }\end{array}$ & Leaves & $\mathrm{S}, \mathrm{C}$ & Oral & $2(4 \%)$ \\
\hline \multicolumn{6}{|l|}{ Combretaceae } \\
\hline Terminalia schimperiana & $\begin{array}{l}\text { Tuit } \\
\text { Idi }\end{array}$ & Leaves & $\mathrm{T}, \mathrm{W}$ & Oral & $4(8 \%)$ \\
\hline \multicolumn{6}{|l|}{ Cucurbitaceae } \\
\hline Citrullus lanatus & $\begin{array}{l}\text { Water melon } \\
\text { Egusi bara }\end{array}$ & Seeds & $\mathrm{H}, \mathrm{C}$ & Oral & $1(2 \%)$ \\
\hline \multicolumn{6}{|l|}{ Cucurbitaceae } \\
\hline Cucurneropsis mannii & $\begin{array}{l}\text { white seed melon } \\
\text { Egusi-itoo }\end{array}$ & Seeds & $\mathrm{H}, \mathrm{C}$ & Oral & $1(2 \%)$ \\
\hline \multicolumn{6}{|l|}{ Cucurbitaceae } \\
\hline Mormodica charantia & $\begin{array}{l}\text { African-cucumber } \\
\text { Ejinrin wewe }\end{array}$ & Leaves & $\mathrm{H}, \mathrm{W}$ & Oral & $5(10 \%)$ \\
\hline \multicolumn{6}{|l|}{ Euphorbiaceae } \\
\hline Jatropha curcas & $\begin{array}{l}\text { Gig nut plant } \\
\text { Batuje }\end{array}$ & Leaves & $\mathrm{S}, \mathrm{C} / \mathrm{W}$ & Oral & $1(2 \%)$ \\
\hline \multicolumn{6}{|l|}{ Euphorbiaceae } \\
\hline Phyllanthus amarus & $\begin{array}{l}\text { Sleeping plant } \\
\text { Ehinolobe }\end{array}$ & Leaves & $\mathrm{H}, \mathrm{C} / \mathrm{W}$ & Oral & $2(4 \%)$ \\
\hline \multicolumn{6}{|l|}{ Fabaceae } \\
\hline Crudia klainei & $\begin{array}{l}\text { Wind tree } \\
\text { Afomo }\end{array}$ & Leaves & $\mathrm{CL}, \mathrm{W}$ & Oral & $2(4 \%)$ \\
\hline \multicolumn{6}{|l|}{ Fabaceae } \\
\hline Tetrapleura tetraptera & $\begin{array}{l}\text { Aidan tree } \\
\text { Arindan }\end{array}$ & Roots & $\mathrm{T}, \mathrm{W}$ & Oral & $2(4 \%)$ \\
\hline \multicolumn{6}{|l|}{ Guttiferae } \\
\hline Garcinia kola & $\begin{array}{l}\text { Bitter kola } \\
\text { Orogbo }\end{array}$ & Roots & $\mathrm{H}, \mathrm{C}$ & Oral & $4(8 \%)$ \\
\hline \multicolumn{6}{|l|}{ Laminaeae } \\
\hline Ocimum gratissimum & Tea bush & Leaves & $\mathrm{H}, \mathrm{C}$ & Oral & $10(20 \%)$ \\
\hline
\end{tabular}




\begin{tabular}{|c|c|c|c|c|c|}
\hline & Efinrin & & & & \\
\hline \multicolumn{6}{|l|}{ Leguminosae } \\
\hline Cassia siamea & $\begin{array}{l}\text { Cassia } \\
\text { Kasia }\end{array}$ & Leaves & $\mathrm{T}, \mathrm{W}$ & Oral & $1(2 \%)$ \\
\hline \multicolumn{6}{|l|}{ Lythraceae } \\
\hline Lawsonia inermis & $\begin{array}{l}\text { Henna } \\
\text { Laali }\end{array}$ & Leaves & $\mathrm{T}, \mathrm{W}$ & Oral & $1(2 \%)$ \\
\hline \multicolumn{6}{|l|}{ Malvaceae } \\
\hline Hibiscus sabdariffa & $\begin{array}{l}\text { Roselle } \\
\text { Isapa }\end{array}$ & Leaves & $\mathrm{H}, \mathrm{C}$ & Oral & $2(4 \%)$ \\
\hline \multicolumn{6}{|l|}{ Malvaceace } \\
\hline Gossipium barbadense & $\begin{array}{l}\text { Cotton } \\
O w u \text {-akese }\end{array}$ & Leaves & $\mathrm{S}, \mathrm{C}$ & Oral & $4(8 \%)$ \\
\hline \multicolumn{6}{|l|}{ Malvaceae } \\
\hline Sida acuta & $\begin{array}{l}\text { Were weed } \\
\text { Esekutu }\end{array}$ & Leaves & $\mathrm{H}, \mathrm{W}$ & Oral & $1(2 \%)$ \\
\hline \multicolumn{6}{|l|}{ Moraceae } \\
\hline Ficus asperifolia & $\begin{array}{l}\text { Sound paper tree } \\
\text { Ipin }\end{array}$ & Leaves & $\mathrm{T}, \mathrm{W}$ & Oral & $1(2 \%)$ \\
\hline \multicolumn{6}{|l|}{ Musaceae } \\
\hline Musa paradisiaaca & $\begin{array}{l}\text { Plantain } \\
\text { Ogede agbaagba }\end{array}$ & Stems & $\mathrm{G}, \mathrm{C}$ & Oral & $4(8 \%)$ \\
\hline \multicolumn{6}{|l|}{ Musaceae } \\
\hline Musa nana & $\begin{array}{l}\text { Banana } \\
\text { Ogede wewe }\end{array}$ & Stems & G, C & Oral & $1(2 \%)$ \\
\hline \multicolumn{6}{|l|}{ Myrtaceae } \\
\hline Syzgyium guineense & $\begin{array}{l}\text { Snake bean tree } \\
\text { Ori }\end{array}$ & Seeds & $\mathrm{T}, \mathrm{W}$ & Oral & $2(4 \%)$ \\
\hline \multicolumn{6}{|l|}{ Phllanthaceae } \\
\hline Bidens pilosa & $\begin{array}{l}\text { black jack } \\
\text { Abere }\end{array}$ & Seeds & $\mathrm{S}, \mathrm{W} / \mathrm{C}$ & Oral & $1(2 \%)$ \\
\hline \multicolumn{6}{|l|}{ Poaceae } \\
\hline Azadirachta indica & $\begin{array}{l}\text { Neem } \\
\text { Dogoyaro }\end{array}$ & Leaves & $\mathrm{T}, \mathrm{W} / \mathrm{C}$ & Oral & $22(44 \%)$ \\
\hline \multicolumn{6}{|l|}{ Meliaceae } \\
\hline Bambus vulgaris & $\begin{array}{l}\text { Bamboo } \\
\text { Oparun }\end{array}$ & Leaves & $\mathrm{S}, \mathrm{C} / \mathrm{W}$ & Oral & $3(6 \%)$ \\
\hline \multicolumn{6}{|l|}{ Poaceae } \\
\hline Cymbopogon citrates & $\begin{array}{l}\text { Lemon grass } \\
\text { Waapa }\end{array}$ & Leaves & G, C & Oral & $1(2 \%)$ \\
\hline \multicolumn{6}{|l|}{ Poaceae } \\
\hline Sorghum caudatum & $\begin{array}{l}\text { Sorghum } \\
\text { Oka baba }\end{array}$ & Shafts & $\mathrm{G}, \mathrm{C}$ & Oral & $1(2 \%)$ \\
\hline \multicolumn{6}{|l|}{ Polygalaceae } \\
\hline $\begin{array}{l}\text { Securidaca } \\
\text { longipedunculata }\end{array}$ & $\begin{array}{l}\text { Violet tree } \\
\text { Ipeta }\end{array}$ & Roots & $\mathrm{T}, \mathrm{W}$ & Oral & $1(2 \%)$ \\
\hline \multicolumn{6}{|l|}{ Rutaceae } \\
\hline Morinda lucida & $\begin{array}{l}\text { Brimstone tree } \\
\text { Owuro }\end{array}$ & Leaves & $\mathrm{T}, \mathrm{W}$ & Oral & $4(8 \%)$ \\
\hline \multicolumn{6}{|l|}{ Rutaceae } \\
\hline Citrus aurantifolia & $\begin{array}{l}\text { Lime } \\
\text { Osanwewe }\end{array}$ & Fruits & $\mathrm{T}, \mathrm{C}$ & Oral & $5(10 \%)$ \\
\hline \multicolumn{6}{|l|}{ Sapindaceae } \\
\hline Bighia sapida & $\begin{array}{l}\text { Akee apple } \\
\text { Isin }\end{array}$ & Leaves & $\mathrm{T}, \mathrm{W} / \mathrm{C}$ & Oral & $3(6 \%)$ \\
\hline \multicolumn{6}{|l|}{ Solanaceae } \\
\hline Nicotiana tabacum & $\begin{array}{l}\text { Tobacco } \\
\text { Taba }\end{array}$ & Leaves & $\mathrm{H}, \mathrm{C}$ & Oral & $1(2 \%)$ \\
\hline \multicolumn{6}{|l|}{ Solanaceae } \\
\hline Solanum aethiopicum & $\begin{array}{l}\text { Mack tomato } \\
\text { Osun }\end{array}$ & Leaves & $\mathrm{H}, \mathrm{W}$ & Oral & $1(2 \%)$ \\
\hline Tiliaceace & & & & & \\
\hline
\end{tabular}




\begin{tabular}{|l|l|l|l|l|l|}
\hline Glyphaea brevis & $\begin{array}{l}---- \\
\text { Atori }\end{array}$ & Leaves & S, W & Oral & $1(2 \%)$ \\
\hline Zingiberaceae & & & & & \\
\hline Aframomum melgueta & $\begin{array}{l}\text { Alligator pepper } \\
\text { Atare }\end{array}$ & Leaves & H, C & Oral & $2(4 \%)$ \\
\hline Zingiberaceae & & & & & \\
\hline Zingiber officinale & $\begin{array}{l}\text { Ginger } \\
\text { Atale }\end{array}$ & Rhizomes & H, C & Oral & $2(4 \%)$ \\
\hline
\end{tabular}

\section{Key}

No of plant species $=50$, No of families $=30, T$-Tree, S-Shrub, H-Herb, G- Grass, CL-Climber. C-Cultivated, w-wild, W/CWild and Cultivated.

Table 3: Some plants species used and their mode of preparation and administration in Abeokuta, Ogun State, Nigeria

\begin{tabular}{|c|c|c|c|c|c|}
\hline No & $\begin{array}{l}\text { Plant species singly or } \\
\text { combination }\end{array}$ & Preparation type & Solvent & Preparation & $\begin{array}{ll}\text { Mode } & \text { of } \\
\text { administration }\end{array}$ \\
\hline 1 & Vernonia amygdalina & $\begin{array}{l}\text { Macerated juice is mixed with } \\
\text { salt }\end{array}$ & Water & Juice extract & $\begin{array}{l}20-25 \text { tablespoonfuls } \\
\text { of the preparation is } \\
\text { taken every morning. }\end{array}$ \\
\hline 2 & Momordica charantia & Boil leaves in water. & Water & Decoction & $\begin{array}{l}\text { About } 350 \mathrm{ml} \text { of the } \\
\text { preparation is taken } \\
\text { daily. }\end{array}$ \\
\hline 3 & $\begin{array}{l}\text { Allium sativum, } \\
\text { Aframomum melegueta, } \\
\text { Carica papaya }\end{array}$ & $\begin{array}{l}\text { Grind dried leaves of } C \text {. papaya, } \\
\text { bulb of } A \text {. sativum and root of } A \text {. } \\
\text { melegueta. }\end{array}$ & Pap & Powder & $\begin{array}{l}\text { A cup-full of the } \\
\text { preparation with hot } \\
\text { pap is taken daily. }\end{array}$ \\
\hline 4 & $\begin{array}{l}\text { Rauwolfia vomitoria } \\
\text { Cirus aurantifolia }\end{array}$ & $\begin{array}{l}\text { Macerate Rauwolfia vomitoria } \\
\text { leaves in water mix with } C \text {. } \\
\text { aurantifolia juice and boil for } 10 \\
\text { minutes. }\end{array}$ & Water & Decoction & $\begin{array}{l}\text { A cup-full }(370 \mathrm{ml}) \text { of } \\
\text { the preparation is } \\
\text { taken daily. }\end{array}$ \\
\hline 5 & Solanum aethiopicum & Macerate leaves in hot water. & Water & Infusion & $\begin{array}{l}\text { The preparations are } \\
\text { taken with glass cup- } \\
\text { full, thrice daily }\end{array}$ \\
\hline 6 & $\begin{array}{l}\text { Saccharum officinarum } \\
\text { Morinda lucida }\end{array}$ & $\begin{array}{l}\text { Macerate fresh leaves of } S . \\
\text { officinarum and } M . \text { lucida then } \\
\text { mix in water. }\end{array}$ & Water & Juice & $\begin{array}{l}\text { Take a cup-full of the } \\
\text { preparation daily. }\end{array}$ \\
\hline 7 & Lawsonia inermis & $\begin{array}{l}\text { Boil fresh leaves of } L . \text { inermis and } \\
\text { potash with water. }\end{array}$ & Water & Decoction & $\begin{array}{l}1 \text { cup-full }(350 \mathrm{ml}) \text { of } \\
\text { the decoction is taken } \\
\text { every morning for } 2 \\
\text { weeks. }\end{array}$ \\
\hline 8 & Jatropha curcas & Burn the fruits into ashes. & Pap & Powder & $\begin{array}{l}\text { Taken a full-cup of the } \\
\text { preparation with pap } \\
\text { every morning before } \\
\text { break- fast. }\end{array}$ \\
\hline 9 & $\begin{array}{l}\text { Bidens pilosa, } \\
\text { Vernonia amygdalina } \\
\text { Momordica charantia } \\
\text { Ocimum gratissimum }\end{array}$ & $\begin{array}{l}\text { Soak B.pilosa seeds, } \\
\text { V.amygdalina leaves, } M \text {. charantia } \\
\text { fruits, and } O \text {. gratissimum leaves } \\
\text { in local gin for } 2 \text { to } 3 \text { days. }\end{array}$ & Gin & Essence & $\begin{array}{l}\text { About }(350 \mathrm{ml}) \text { of the } \\
\text { preparation is taken } \\
\text { every morning. }\end{array}$ \\
\hline 10 & $\begin{array}{l}\text { Vernonia amygdalina } \\
\text { Bidens pilosa } \\
\text { Uvaria afzelii }\end{array}$ & $\begin{array}{l}\text { Soak leaf, seeds and root of } V . \\
\text { amygdalina, B. pilosa and } U \text {. } \\
\text { afzelii root for } 2 \text { to } 3 \text { days. . }\end{array}$ & Water & Maceration & $\begin{array}{l}\text { A cup-full of the } \\
\text { extracts is drank thrice } \\
\text { daily. }\end{array}$ \\
\hline 11 & $\begin{array}{l}\text { Vernonia amygdalina } \\
\text { Allium cepa }\end{array}$ & $\begin{array}{l}\text { Soak leaves } V . \text { amygdalina and } \\
\text { bulb of } A \text {. cepa in water for } 4 \text { days. }\end{array}$ & Water & Maceration & $\begin{array}{l}\text { 1 cup-full } \\
400 \mathrm{ml}) \text { of } \\
\text { preparation is the } \\
\text { daily. }\end{array}$ \\
\hline 12 & Carica papaya & $\begin{array}{l}\text { Boil unripe fruits of } C \text {. papaya in } \\
\text { water. }\end{array}$ & Water & Decoction & $\begin{array}{l}25 \text { tablespoonfuls of } \\
\text { the preparation are } \\
\text { taken daily. }\end{array}$ \\
\hline 13 & $\begin{array}{l}\text { Anana comosus } \\
\text { Carica papaya } \\
\text { Ficus asperfolia } \\
\end{array}$ & $\begin{array}{l}\text { Boil Fruits of } A \text {. comosus, unripe } \\
\text { fruit of } C \text {.papaya and leaves of } F \text {. } \\
\text { asperfolia in water. }\end{array}$ & Water & Decoction & $\begin{array}{l}\text { A cup-full of the } \\
\text { extracts is drank twice } \\
\text { daily }\end{array}$ \\
\hline 14 & $\begin{array}{l}\text { Cucumeropsis mannii } \\
\text { Citrus aurantifolia }\end{array}$ & $\begin{array}{l}\text { Mix the fruit juice of } \\
\text { Cucumeropsis mannii }\end{array}$ & Lime & Juice & $\begin{array}{l}20-25 \text { tablespoonfuls } \\
\text { of the preparation are }\end{array}$ \\
\hline
\end{tabular}




\begin{tabular}{|c|c|c|c|c|c|}
\hline & & and $C$. aurantifolia in potash. & & & $\begin{array}{l}\text { taken in the morning } \\
\text { or at night. }\end{array}$ \\
\hline 15 & Bidens pilosa & $\begin{array}{l}\text { Grind dried roots into powder, mix } \\
\text { in hot pap and honey. }\end{array}$ & $\begin{array}{l}\text { Honey/ } \\
\text { pap }\end{array}$ & Powder & $\begin{array}{l}25 \text { tablespoonfuls are } \\
\text { taken daily }\end{array}$ \\
\hline 16 & $\begin{array}{l}\text { Vernonia amygdalina } \\
\text { and Glyphaea brevis }\end{array}$ & $\begin{array}{l}\text { Boil Leaves of } V \text {. amygdalina and } \\
G . \text { brevis in water for } 20 \mathrm{~min} \text {. }\end{array}$ & Water & Decoction & $\begin{array}{l}\text { A cup-full of the } \\
\text { preparation is taken } \\
\text { before break- fast } \\
\text { daily. }\end{array}$ \\
\hline 17 & Citrullus lanatus & $\begin{array}{l}\text { Boil fruit of C. lanatus and potash } \\
\text { with water. }\end{array}$ & Water & Decoction & $\begin{array}{l}1 \text { cup is drunk every } 3 \\
\text { days. }\end{array}$ \\
\hline 18 & $\begin{array}{l}\text { Allium sativum } \\
\text { Vernonia amygdalina } \\
\text { Ocimum gratissimum }\end{array}$ & $\begin{array}{l}\text { Boil bulb of } A \text {. sativum, leaves of } \\
V \text {.amygdalina, and } O \text {. gratissimum } \\
\text { and potash in water. }\end{array}$ & Water & Decoction & $\begin{array}{l}\text { Take a cup-full } \\
(350 \mathrm{ml}) \text { daily before } \\
\text { break- fast. }\end{array}$ \\
\hline 19 & $\begin{array}{l}\text { Allium cepa and } \\
\text { Carica papaya } \\
\text { Aframomum melegueta }\end{array}$ & $\begin{array}{l}\text { Grind into powder dried bulb of } \\
\text { A. cepa, root of } C \text {. papaya and } \\
\text { seeds of } A \text {. melegueta. }\end{array}$ & Pap & Powder & $\begin{array}{l}\text { The powder form of } \\
\text { the plants sample is } \\
\text { taken with hot pap. }\end{array}$ \\
\hline 20 & $\begin{array}{l}\text { Securidaca } \\
\text { longipedunculata }\end{array}$ & $\begin{array}{l}\text { Soak root of } S \text {. longipedunculata } \\
\text { in local gin for } 3 \text { days. }\end{array}$ & Gin & Essence & $\begin{array}{l}1 \text { cup-full of the } \\
\text { extracts is taken every } \\
3 \text { days. }\end{array}$ \\
\hline 21 & $\begin{array}{l}\text { Nicotiana tabacum } \\
\text { Bidens pilosa } \\
\text { Alstonia congensis }\end{array}$ & $\begin{array}{l}\text { Boil with water } N \text {. tabacum leaves, } \\
\text { B. pilosa seeds, } \\
\text { A. congensis root and potash. }\end{array}$ & Water & Decoction & $\begin{array}{l}1 \text { cup-full } 350- \\
400 \mathrm{ml}) \text { of decoction is } \\
\text { taken every } 3 \text { days } \\
\text { interval before break- } \\
\text { fast. }\end{array}$ \\
\hline 22 & $\begin{array}{l}\text { Uvaria afzelii } \\
\text { Securidaca } \\
\text { longipedunculata }\end{array}$ & $\begin{array}{l}\text { Grind into powder form root and } \\
\text { bark of Securidaca } \\
\text { longipedunculata and U. afzelii. }\end{array}$ & Pap & Powder & $\begin{array}{l}\text { The powder are mixed } \\
\text { with hot pap and taken } \\
\text { twice daily. }\end{array}$ \\
\hline 23 & Jatropha curcus & $\begin{array}{l}\text { Boil Leaves of } J . \quad \text { Curcas and } \\
\text { palm oil with water. }\end{array}$ & Water & Decoction & $\begin{array}{l}1 \text { cup-full of the } \\
\text { preparation is taken } \\
\text { every morning. }\end{array}$ \\
\hline 24 & Citrus aurantifolia & $\begin{array}{l}\text { Mix } C \text {. aurantifolia fruit juice and } \\
\text { potash with cow bile. }\end{array}$ & $\begin{array}{l}\text { Cow } \\
\text { bile }\end{array}$ & Juice & $\begin{array}{l}18 \text { tablespoonfuls of } \\
\text { juice are taken every } 3 \\
\text { days. }\end{array}$ \\
\hline 25 & Ficus asperifolia & $\begin{array}{l}\text { Boil } F \text {. asperifolia root and C. } \\
\text { aurantifolia juice in water. }\end{array}$ & Water & Decoction & $\begin{array}{l}400 \mathrm{ml} \text { of the } \\
\text { preparation is taken } \\
\text { daily before break- } \\
\text { fast. }\end{array}$ \\
\hline 26 & Musa nana & $\begin{array}{l}\text { Mix Juice of flower of } M . \text { nana } \\
\text { with honey. }\end{array}$ & Honey & Juice & $\begin{array}{l}20 \text { tablespoonfuls are } \\
\text { taken daily. }\end{array}$ \\
\hline 27 & Musa parasidiaca & $\begin{array}{l}\text { Soak Stems of } M \text {. parasidiaca in } \\
\text { water for } 2 \text { to } 3 \text { days. }\end{array}$ & Water & Maceration & $\begin{array}{l}21 \text { tablespoonfuls of } \\
\text { macerate are taken } \\
\text { daily. }\end{array}$ \\
\hline 28 & Garcinia kola & $\begin{array}{l}\text { Boil roots of } G \text {. kola with water } \\
\text { for } 1 \text { hour }\end{array}$ & Water & Decoction & $\begin{array}{l}1 \text { cup-full }(350 \mathrm{ml}) \text { is } \\
\text { taken every } 3 \text { days } \\
\text { interval. }\end{array}$ \\
\hline 29 & $\begin{array}{l}\text { Zingiber officinale } \\
\text { Allium sativum }\end{array}$ & $\begin{array}{l}\text { Boil rhizomes of } Z \text {. officinale and } \\
\text { bulbs of } A \text {. sativum in hot water. }\end{array}$ & Water & Decoction & $\begin{array}{l}1 \text { cup-full }(350 \mathrm{ml}) \text { of } \\
\text { herbal preparation is } \\
\text { taken twice daily. }\end{array}$ \\
\hline 30 & $\begin{array}{l}\text { Azardirachta indica } \\
\text { Anacardum occidentale }\end{array}$ & $\begin{array}{l}\text { Boil leaves of } A \text {. indica and } A \text {. } \\
\text { occidentales with water. }\end{array}$ & Water & Decoction & $\begin{array}{l}25 \text { tablespoonfuls are } \\
\text { taken at night. }\end{array}$ \\
\hline 31 & Gossipium spp. & Boil leaves of Gossipium in water. & Water & Decoction & $\begin{array}{l}20 \text { tablespoonfuls of } \\
\text { the decoction is taken } \\
\text { twice daily. }\end{array}$ \\
\hline 32 & Bambus vulgaris & $\begin{array}{l}\text { Soak leaves of B. vulgaris in cold } \\
\text { water for } 3 \text { days }\end{array}$ & Water & Macerate & $\begin{array}{l}\text { The cold extract is } \\
\text { taken every morning } \\
\text { for six weeks. }\end{array}$ \\
\hline 33 & $\begin{array}{l}\text { Azadirachta indica } \\
\text { Garcinia kola }\end{array}$ & $\begin{array}{l}\text { Boil seeds of A. indica and G. kola } \\
s p \text { in water for } 1_{1 / 2} \text { hours. }\end{array}$ & Water & Decoction & $\begin{array}{l}1 \text { cup-full }(330 \mathrm{ml}) \text { is } \\
\text { taken every day before } \\
\text { eating. }\end{array}$ \\
\hline 34 & $\begin{array}{l}\text { Garcinial cola } \\
\text { Cymbopogon citrates }\end{array}$ & $\begin{array}{l}\text { Macerate juice of G. kola sp and } \\
\text { Cymbopogon citratus leaves in }\end{array}$ & Water & Juice & $\begin{array}{l}20 \text { tablespoonfuls of } \\
\text { juice are taken daily. }\end{array}$ \\
\hline
\end{tabular}




\begin{tabular}{|c|c|c|c|c|c|}
\hline & & little amount of water. & & & \\
\hline 35 & Cassia siamea & $\begin{array}{l}\text { Boil C. siamea leaves in water for } \\
1 \mathrm{hr} \text {. }\end{array}$ & Water & Decoction & $\begin{array}{l}1 \text { cup-full of decoction } \\
\text { is taken every } 2 \text { days. }\end{array}$ \\
\hline 36 & Gossipium spp. & $\begin{array}{l}\text { Boil roots/ leaves of Gossipium in } \\
\text { water for } 1 \text { hour. }\end{array}$ & Water & Decoction & $\begin{array}{l}\text { The decoction is taken } \\
\text { daily. }\end{array}$ \\
\hline 37 & $\begin{array}{l}\text { Azadirachta indica } \\
\text { Lawsonia inermis }\end{array}$ & $\begin{array}{l}\text { Boil leaves of A. indica and } \\
\text { Lawsonia inermis in water for } 1 \mathrm{hr}\end{array}$ & Water & Decoction & $\begin{array}{l}\text { A cup-full of the } \\
\text { preparations is taken } \\
\text { every day. }\end{array}$ \\
\hline 38 & $\begin{array}{l}\text { Azadirachta indica } \\
\text { Ageratum conyzoides }\end{array}$ & $\begin{array}{l}\text { Boil leaves of } A . \text { indica and } A \text {. } \\
\text { conyzoides in water for } 1 \text { hour. }\end{array}$ & Water & Decoction & $\begin{array}{l}20-22 \text { tablespoonfuls } \\
\text { of the decoction is } \\
\text { taken twice daily } \\
\text { before break- fast. }\end{array}$ \\
\hline 39 & $\begin{array}{l}\text { Terminalia } \\
\text { schimperiana }\end{array}$ & $\begin{array}{l}\text { Soak leaves of } T \text {. schimperiana } \\
\text { and Gossipium in water for 2- } \\
\text { 3days. }\end{array}$ & Water & Maceration & $\begin{array}{l}340 \mathrm{ml} \text { of the extract is } \\
\text { taken daily. }\end{array}$ \\
\hline 40 & Azadirachta indica & $\begin{array}{l}\text { Grind into powder fruits of } A \text {. } \\
\text { indica. }\end{array}$ & Pap & Powder & $\begin{array}{l}\text { The powder of the is } \\
\text { drunk with hot pap } \\
\text { daily }\end{array}$ \\
\hline 41 & Cassia siamea & $\begin{array}{l}\text { Macerate Leaves of Cassia } \\
\text { siamea } \text { water. }\end{array}$ & Water & Infusion & $\begin{array}{l}18-22 \text { tablespoonfuls } \\
\text { of the preparation are } \\
\text { taken daily. }\end{array}$ \\
\hline 42 & Azadirachta indica & $\begin{array}{l}\text { Soak in local gin, leaves of } \\
\text { Azadirachta indica indica for } 2 \text { to } \\
3 \text { days. . }\end{array}$ & Gin & Essence & $\begin{array}{l}1 \text { cup-full of the } \\
\text { decoction is taken } \\
\text { daily. }\end{array}$ \\
\hline 43 & $\begin{array}{l}\text { Gossipium sp. } \\
\text { Ocimum gratissimum }\end{array}$ & $\begin{array}{l}\text { Boil leaves of Gossipium and } O \text {. } \\
\text { gratissimum in water. }\end{array}$ & Water & Decoction & $\begin{array}{l}\text { A cup-full }(400 \mathrm{ml}) \text { of } \\
\text { the extract is drunk } \\
\text { before break-fast } \\
\text { daily. }\end{array}$ \\
\hline 44 & $\begin{array}{l}\text { Mangifera indica } \\
\text { Terminalia } \\
\text { schimperianae }\end{array}$ & $\begin{array}{l}\text { Soak leaves of } M . \text { indica } T . \\
\text { schimperianae in water for } 3 \text { days. }\end{array}$ & Water & Macerate & $\begin{array}{l}\text { The cold preparation } \\
\text { is taken daily every } \\
\text { morning. }\end{array}$ \\
\hline 45 & Cassia siamen & $\begin{array}{l}\text { Soak bark of } C \text {. siamen in local gin } \\
\text { for } 2 \text { days. }\end{array}$ & Gin & Essence & $\begin{array}{l}20 \text { tablespoonfuls are } \\
\text { taken every 3days. }\end{array}$ \\
\hline 46 & Morinda lucida & $\begin{array}{l}\text { Grind bark of } M \text {. lucida into } \\
\text { powder and take with pap. }\end{array}$ & Pap & Powder & $\begin{array}{l}\text { The powder is mixed } \\
\text { with pap. Drink daily. }\end{array}$ \\
\hline 47 & $\begin{array}{l}\text { Tridax procumbens } \\
\text { Rauwolfia vomitoria }\end{array}$ & $\begin{array}{l}\text { Boil leaves of } T \text {. procumbens and } \\
\text { Rauwolfia vomitoria in water. }\end{array}$ & Water & Decoction & $\begin{array}{l}\text { The decoction is taken } \\
\text { every morning for at } \\
\text { least } 6 \text { weeks. }\end{array}$ \\
\hline 48 & Zingiber officinale & $\begin{array}{l}\text { Soak rhizomes of } Z \text {. officinale in } \\
\text { local gin for } 3 \text { days. }\end{array}$ & Gin & Essence & $\begin{array}{l}1 \text { cup-full }(300- \\
320 \mathrm{ml}) \text { of essence is } \\
\text { taken. }\end{array}$ \\
\hline 49 & Azadirachta indica & $\begin{array}{l}\text { Boil Seeds and leaves of A. indica } \\
\text { in water for one hour. }\end{array}$ & Water & Decoction & $\begin{array}{l}\text { A full-cup }(340 \mathrm{ml}) \text { of } \\
\text { the preparation is } \\
\text { taken every morning } \\
\text { before break- fast. }\end{array}$ \\
\hline 50 & $\begin{array}{l}\text { Terminalia } \\
\text { schimperiana }\end{array}$ & Chew roots of $T$. schimperiana. & Saliva & Juice & $\begin{array}{l}\text { The root is chewed } \\
\text { every morning for one } \\
\text { month. }\end{array}$ \\
\hline
\end{tabular}

Table 4: Life forms, habitat, plant parts used, solvents, dosage measurement and non- plant materials used in treating diabetes mellitus

\begin{tabular}{|l|l|l|l|}
\hline Plant forms & Frequency & \% of total & Mode \\
\hline Tree & & & \\
\hline Shrubs & 16 & 32 & \\
\hline Climber & 7 & 14 & \\
\hline Herbs & 1 & 2 & 42 \\
\hline Total & 26 & 52 & \\
\hline Habitat & $\mathbf{5 0}$ & $\mathbf{1 0 0}$ & \\
\hline
\end{tabular}




\begin{tabular}{|c|c|c|c|}
\hline Cultivated & 23 & 46 & 46 \\
\hline Wild & 18 & 36 & \\
\hline Both & 9 & 18 & \\
\hline Total & 50 & 100 & \\
\hline \multicolumn{3}{|l|}{ Commonly used plant parts } & \\
\hline Root & 5 & 10 & \\
\hline Leaf/root & 1 & 2 & \\
\hline Leaf/bark & 1 & 2 & \\
\hline Leaf & 31 & 62 & 62 \\
\hline Seed & 4 & 8 & \\
\hline Fruit & 2 & 4 & \\
\hline Rhizome & 1 & 2 & \\
\hline Shaft & 1 & 2 & \\
\hline Stem (Culm) & 2 & 4 & \\
\hline Bulb & 2 & 4 & \\
\hline Total & 50 & 100 & \\
\hline \multicolumn{4}{|l|}{ Common preparation methods } \\
\hline Juice & 7 & 14 & \\
\hline Decoction & 23 & 46 & 46 \\
\hline Powder & 7 & 14 & \\
\hline Infusion & 2 & 4 & \\
\hline Essence & 5 & 10 & \\
\hline Macerate & 6 & 12 & \\
\hline Total & $\mathbf{5 0}$ & 100 & \\
\hline \multicolumn{4}{|l|}{ commonly used solvents } \\
\hline Water & 32 & 64 & 64 \\
\hline Pap & 6 & 12 & \\
\hline Gin & 6 & 12 & \\
\hline Lime Juice & 2 & 4 & \\
\hline Honey & 2 & 4 & \\
\hline Bile of cow & 1 & 2 & \\
\hline Saliva & 1 & 2 & \\
\hline Total & $\mathbf{5 0}$ & 100 & \\
\hline \multicolumn{4}{|c|}{ Daily dosage measurement of herbal preparation } \\
\hline Tablet & 0 & 0 & \\
\hline Capsule & 1 & 2 & \\
\hline Cups & 38 & 76 & 76 \\
\hline Drops & 0 & 0 & \\
\hline Total & $\mathbf{5 0}$ & 100 & \\
\hline Teaspoons & 11 & 22 & \\
\hline \multicolumn{4}{|l|}{ Duration of usage } \\
\hline Weeks & 10 & 20 & \\
\hline Months & 36 & 72 & \\
\hline Years & 4 & 8 & \\
\hline Total & 50 & 100 & \\
\hline \multicolumn{4}{|l|}{ Other recommendations } \\
\hline Non - plan material recommended & & 4 & \\
\hline Beans and its derivatives & 13 & 26 & \\
\hline vegetables and fruits & 30 & 60 & 60 \\
\hline No diet & 5 & 10 & \\
\hline
\end{tabular}

\section{DISCUSSION}

The observation that larger percentage of respondent (Traditional herbal practitioners) was males could be an indication that males involved in herbal practice than their female counterparts. This is contrary to the finding of Durmuşkahya and Öztürk (2013) who reported that women aged between 39 and 61 years use much more herbal plants than men. Unlike in the olden days when traditional practitioners were illiterates and consequently could not document the observation of (c) 2011-15, JDDT. All Rights Reserved their herbal practices hence, the present study have shown that majority of the respondents were educated. The basic education attainment enhanced them the necessary proficiency needed for documentation of their herbal practice. Similar observation was reported by Kadiri et al., (2013); Durmuşkahya and Öztürk, (2013). Islam and Christianity were the predominant religions of the respondents. This is an indication that herbal practice is not affected by any religion affiliation. 
The traditional healers consulted in the study claimed to have detected diabetes mellitus in their patients by observing symptoms such as weight loss, fatigue and excessive or frequent urination. The practitioners also claimed to have treated diabetes for up to 3-35years, having acquired the knowledge mainly through training and parental inheritance. Some of the respondents claimed to use mainly herbs while nonplant materials such as honey, local gin and minerals like alum and potash were also recommended in combination with plant materials. These results are in line with findings of Adebayo, (2009).

Most diabetic patients interviewed were between the 20-70 years, indicating predominance of type-2 diabetes (Jouad et al., 2001; Sobngwi et al., 2001). The preparations were claimed to have been used successfully for the treatment of diabetes for weeks, months and years depending on the severity of the disease without side effects in any of the plants.

The plant families with the highest occurrence of species include asteraceae and apocynaceae with 4 species respectively followed by Cucurbitaceae, malvaceae and poaceae having 3 species in that order. The frequent occurrence of other families also suggests their importance as repository of useful plants which may be explored for treatment of diabetes and other diseases.

The most frequently employed life form of plants covered by this study was herbs, followed by tress. Similar observation was reported by Raut et al., (2012).

Results also revealed that 50 traditional medicinal plants were used by people of Abeokuta for the treatment of diabetes. The observation that leaves were reported as the most frequently used plant parts is in conformity with the finding of Pratul, (2011); Koumba Madingou et al.,(2012) Manishayadav et al., (2012); Raut et al., (2013): Vinatha and Estari, (2013). This observation may be an indication that leaves are major site of deposition of plant secondary metabolites.

It is difficult to say precisely which method of preparation is most commonly accepted because it is different from one therapeutist to another. According to Audrey and Dehin (2004), extraction of active ingredients is made by boiling the plant while Thomson (1981) reported that preparation methods

\section{REFERENCES}

1. Adebayo, A. G. (2009). Inventory of antidiabetic plants in selected districts of Lagos State, Nigeria. Journal of Ethnopharmacology 121: 135-139

2. Adekunle, V. A. J. and Akinlemibola, O. (2008). Effect of deforestation on climate change and global warming in Nigeria. In: Popoola, L. (ed.). Proceedings of the $32{ }^{\text {nd }}$ Annual Conference of Forestry Association of Nigeria (FAN) held in Umuahia, Aba State, Nigeria between 20 and 24 October, Pp 170-182.

3. Aslan, M., Orhan, N., Orhan, D.D. \& Ergun, F. (2010). Hypoglycemic activity and antioxidant potential of some medicinal plants traditionally used in Turkey for diabetes. Journal of Ethnopharmacology 128: 384-389.

4. Aguwa CN. Therapeutic Basis for Clinical Pharmacy in the Tropics. Third edn. SNAAP Press Ltd, Enugu. 2004: 1-230. vary depending on the types of plant. As regard this study, preference was given to boiling in water than soaking in alcohol or honey. This is believed to be more economically efficient and contrary to the report of Idowu et al., (2009). The plant materials prepared as decoction, infusion in milk or honey were used for the treatment of diabetes as also reported by Thirumalai et al.,(2012).

Soaking was preferred by its users as they believe that the ingredients will be extracted without being exposed to heat which may have adverse effect on the efficacy of the preparations. The mode of administration of these different recipes is by oral route $(350-400 \mathrm{ml})$ which is the most used not only in traditional medicine but also in modern medicine. Apart from herbal therapy, appropriate diets such as beans and its derivatives, non-starchy vegetables, fruits and exercises were also recommended by THPs as also reported by Adebayo, (2009).

These plants preparations can be used isolated or in combination as a concoction. The respondent claimed that the recipes are effective despite their mode of preparation.

Majority of the recipes documented in the study were multi - component in nature. Mono - component recipes have also been found to be effective (Haddad $e t$ al 2003: Kareru et al., 2007). Also, combination of plant parts mainly by decoction was reported as most common recipe used in the treatment of diabetes mellitus (Vinatha and Estari, 2013; Koumba Madingou et al., 2012). This may support the reports that herbal preparations are more potent in the treatment of disease when the plant secondary metabolites are in combined form (Dinet et al., 2011; Kadiri et al., 2013; Vinatha and Estari, 2013. The information recorded from herbal healers indicates that they possess good knowledge of anti-diabetic herbal drugs. Documentation of traditional knowledge of local traditional healers is very useful for future generation (Vinatha and Estari, 2013)

\section{CONCLUSION}

This study showcased the use of indigenous flora of Abeokuta used for management of diabetes, hence, sustainable management of the forest is highly advocated.
5. Ceylan, S., Azal, O. and Taslipinar, A. (2009). Complementary and alternative medicine use among Turkish diabetes patients. Complementary Therapies in Medicine 17: 78-83.

6. Din, N., Dibong, S. D. , Mpondo, M.E., Priso, R.J ., Kwin, N.F and Ngoye, A. (2011). Inventory and Identification of Plants Used in the Treatment of Diabetes in Douala Town (Cameroon) European Journal of Medicinal Plants 1(3): 6073 ,

7. Durmuşkahya, C and Öztürk, M. (2013.). Ethnobotanical Survey of Medicinal Plants Used for the Treatment of Diabetes in Manisa, Turkey Sains Malaysiana 42(10): 14311438 .

8. Farnsworth, N.R, Kinghorn, A.D, Soejarto ,D.D, and Waller, D.P. (1985). Siberian Ginseng (Eleutherococcus 
senticosus) current status as an Adaptogen. In: Wagner $\mathrm{H}$, Hikino H, Farnsworth NR (Eds). Economics and Medicinal Plant Research. 1. Academics Press, Orlando, FL. 1: 155 215.

9. Haddad, P.S., Depot, M., Settafa, A., Chabli, A. and Cherrah, Y. (2003) . Comparative study on the medicinal plants most recommended by traditional practitioners in Morcco and Canada. Journal of Herbs, Spices and Medicinal Plants 10:25-45

10. Idowu, O.A., Soniran, O.T., Ajana, O., and Aworinde, D.O. (2009). Ethnobotanical survey of antimalarial plants used in Ogun State, Southwest Nigeria. African Journal of Pharmacy and Pharmacology, 4(2) 055-060,

11. Inanç, N., Çiçek, B. \& Sahin, H. (2007). Use of herbs by the patients with diabetes in Kayseri, Turkey. Pakistan Journal of Nutrition 6: 310-312.

12. Israili, Z.H. (2010). Diabetes and Its Treatment with Botanicals in Turkey. Merkez Efendi Symposium Book, Denizli, Turkey.

13. Israili, Z.H. (2011). Advances in the treatment of type 2 diabetes mellitus. American Journal of Therapeutics 18: 117152.

14. Jouad., Rhiouani, H., EL-Hilaly .J. and Eddouk, M. (2001).Ethnobotanical survey of medicinal plants used for the treatment of diabetes, cardiac and renal disease in north center of Morocco (Fez-Boulenmane). Journal Ethnopharmacology 77: 175-182.

15. Kadiri1 M,Ojewumi A.W, and Adegboye O.O. (2013).Folk use of herbal plants used in the treatment of malaria fever in Abeokuta North Local Government, Ogun, State. Nigeria Direct Research Journal of Health and Pharmacology (DRJHP) .1 (2), 10-19.

16. Kareru, P.G., Kenji, G.M., Gachanja, A.N., Keriko , J.M. and Mungai, G. (2007). Traditional medicine among the Embu and Mbere people of Kenya. African Journal of Traditional Complementary and Alternative Medicine 4: 75-86.

17. Koumba Madingou, N.O., Souza, A , Lamidi, M. L.E. Mengome, L. E Eyele M. M.C.,1, Bading, B., Mavoungou, J ., Traore,A.S. (2013). Study of medicinal plants used in the management of cardiovascular diseases at libreville (gabon): an ethnopharmacological approach., IJPSR, 3(1): 111-119.
18. Küçükgüçlü, Ö., Kızılcı, S., Mert, H., Uğur, Ö., Besen, D.B.and Ünsal, E. (2010). Complementary and alternative medicine use among people with diabetes in Turkey. Western Journal of Nursing Research 22: 20-26.

19. Manishayada V, K., and Beg, M.Z. (2012). Medicinal plants used for the treatment of diabetes by the baiga Tribe living in rewa district m.p. Indian J.L.Sci.2(1) : 99-102,

20. Morris, K. (2002). South Africa tests traditional medicines. Lancet Infect. Dis. 2: 319.

21. Onal, S., Timur, S., Okutucu, B. \& Zihnioğlu, F. (2005). Inhibition of alpha-glucosidase by aqueous extracts of some potent antidiabetic medicinal herbs. Preparative Biochemistry and Biotechnology 35: 29-36.

22. Özbek, H., Ceylan, E., Kara, M., Özgökçe, F. and Koyuncu, M. (2004). Hypoglycemic effect of Rheum ribes roots in alloxan induced diabetic and normal mice. Scand. J. Lab. Anim. Sci. 31(2): 113-115.

23. Pratul, C. S. (2011).Ethno Antidiabetic Plants Of Assam. International journal of applied biology and pharmaceutical technology. 246-251

24. Raut, S., Raut, S,. Sen, S.K., Satpathy, S and Pattnaik, D. (2012). An Ethnobotanical Survey of Medicinal Plants in Semiliguda of Koraput District, Odisha, India. Botany Research International 5 (4): 97-107, 2

25. Soladoye, M. O., Chukwuma, E. C. and Owa, F. P. (2012). An 'Avalanche' of Plant Species for the Traditional Cure of Diabetes mellitus in South-Western Nigeria. J. Nat. Prod. Plant Resour., 2 (1): 60-72

26. Thomson, A. (1981). Medicinal plants: botany and ethnology. Berger-leurault, Paris $206 \mathrm{p}$.

27. Technical Report. Series 646. Word Health Organisation Geneva.(accessed 4/06/2006).

28. Thirumalai, T., Beverly, C. D., Sathiyaraj, K. B.Senthilkumar, E., David, E. (2012). Ethnobotanical Study of Anti-diabetic medicinal plants used by the local people in Javadhu hills Tamilnadu, India Asian Pacific Journal of Tropical Biomedicine S910-S913.

29. Vinatha, N and Estari, D. (2013). An Ethnobotanical Study Of Plants Used For The Treatment Of Diabetes In The Warangal District, Andhra Pradesh, India An International Quarterly Journal Of Biology \& Life Sciences 1(1):-24-28. 